\title{
Experimental Investigation of a SMC High Precision Control
}

\author{
Khalid Abidi* and Asif Şabanoviç** \\ *National University of Singapore, Singapore, \\ **Faculty of Engineering and Natural Sciences, Sabanci University \\ 34956, Tuzla, Istanbul, Turkey \\ khalida@su.sabanciuniv.edu, asif@sabanciuniv.edu
}

\begin{abstract}
In this paper a discrete-time Sliding-Mode (SM) based controller for high accuracy position control is investigated. The controller is designed for a general SISO system with nonlinearity and external disturbance. It will be shown that application of the proposed controller forces the state trajectory to be within an $O\left(T_{s}^{2}\right)$. The proposed controller is applied to a stage driven by a piezo drive that is known to suffer from nonlinearity. As a separate idea to enhance the accuracy of the closed loop system a combination of disturbance rejection method and the SMC controller is explored and its effectiveness is experimentally demonstrated. Closed-loop experiments are presented using PID controller with and without disturbance compensation and Sliding-Mode Controller with and without disturbance compensation for the purpose of comparison.
\end{abstract}

\section{INTRODUCTION}

Sliding mode controllers have proven to be very powerful algorithms in the face of systems with plant parameter variations, unmodeled dynamics and external disturbances. However, design of classical sliding mode controllers requires knowledge of plant dynamics. This is not favorable for cases where partial plant dynamics are known. Hence, it is necessary to derive a controller that can satisfy above mentioned properties while requiring partial knowledge of plant parameters in its design. For the cases in discrete-time sliding mode control switching control is not possible due to the switching frequency being limited by the sampling rate of the controller. Hence, the control must be continuous unlike the case for continuous-time applications where the control is discontinuous in nature. In the work of, [1], the derived sliding mode controller was proven to force the states to be in $O\left(T_{s}^{2}\right)$ boundary around the desired sliding surface. Hence, the proposed controller must be capable of showing the same characteristics. Finally, experiments will be conducted on piezo actuator driven stage.

Piezoelectric actuators have shown a great potential in applications that require sub-micrometer down to nanometer motion. The advantages that piezoelectric actuators offer are the absence of friction and stiction characteristics that exist in other actuators. Thus, piezoelectric actuators are ideal for very high-precision motion applications. The main characteristics of piezoelectric actuators are: extremely high resolution in the nanometer range, high bandwidth up to several kilo hertz range, a large force up to few tons, and very short travel in the sub-millimeter range. Application areas of piezoelectric actuators include: micromanipulation, micro-assembly, add-ons for high precision cutting machinery and as secondary actuators in macro/micro motion systems such as dual-stage hard-disk drives. In all of these applications the accuracy of positioning is very important and in many cases the closed loop control is the only answer. Despite of this there are many attempts to drive piezo stacks as an open loop system with fine compensation of the hysteresis nonlinearity in one or another way. With development of accurate position transducers the possibility to use robust feedback based nonlinear control methods is becoming attractive alternative to the model based compensation.

As a final extension of the work, a disturbance observer based on the lumped parameter model of the piezo-stage proposed in, [2], will be experimentally shown to improve the overall performance of the closed-loop system

\section{CONTROLlER DESIGN AND ANALYSIS}

\section{A. Controller Design}

Consider the general system defined below

$$
\dot{x}=f(x)+B u
$$

Here, it is assumed that $f$ and $B$ are smooth, continuous and bounded. The aim is to drive the states of the system into the set $S$ defined by

$$
S=\left\{x: G\left(x^{r}-x\right)=\sigma\left(x, x^{r}\right)=0\right\}
$$

Here $G$ is a positive constant, $x$ is the state vector, $x_{r}$ is the reference vector and it will be assumed to be smooth and continuous, and $\sigma\left(x, x^{r}\right)$ is the function defining the sliding mode manifold. 
The derivation of the control law starts with the selection of the Lyapunov function, $V(\sigma)$, and an appropriate form of the derivative of the Lyapunov function, $\dot{V}(\sigma)$.

Lyapunov function selection such that it is positive definite

$$
V(\sigma)=\frac{\sigma^{2}}{2}
$$

Hence the derivative of the Lyapunov function is

$$
\dot{V}(\sigma)=\sigma \dot{\sigma}
$$

In order to guaranty the stability of solution $\sigma\left(x, x^{r}\right)=0$, the derivative of the Lyapunov function is selected to be

$$
\dot{V}(\sigma)=-D \sigma^{2}
$$

Here $D$ is a positive constant. Hence, if control can be determined from (4) and (5), the stability of the solution (5) will be guaranteed since $V(\sigma)>0, V(0)=0$ and $\dot{V}(\sigma)<0, \quad \dot{V}(0)=0$. By combining (4) and (5) the following result is obtained

$$
\sigma(\dot{\sigma}+D \sigma)=0
$$

A solution for (6) is as follows

$$
\dot{\sigma}+D \sigma=0
$$

The derivative of the sliding function is as follows

$$
\dot{\sigma}=G\left(\dot{x}^{r}-\dot{x}\right)=G \dot{x}^{r}-G \dot{x}
$$

From (8) and using (1)

$$
\dot{\sigma}=\underbrace{G \dot{x}^{r}-G f}_{G B u_{e q}}-G B u(t)=G B\left(u_{e q}-u(t)\right)
$$

If (9) is inserted in (7) and the result is solved for the control

$$
u(t)=u_{e q}+(G B)^{-1} D \sigma
$$

It can be seen from (9) that $u_{e q}$ is difficult to calculate. Using the fact that $u_{e q}$ is a continuous function since it is a function of $x^{r}$ and $f$ that are assumed smooth and continuous, (9) can be written in discrete-time form after applying Euler's approximation,

$$
\frac{\sigma\left((k+1) T_{s}\right)-\sigma\left(k T_{s}\right)}{T_{s}}=G B\left(u_{e q}\left(k T_{s}\right)-u\left(k T_{s}\right)\right)
$$

Here $T_{s}$ is the sampling time and $k \in Z^{+}$. It is also necessary to write (10) in discrete-time form just as it was done before

$$
u\left(k T_{s}\right)=u_{e q}\left(k T_{s}\right)+(G B)^{-1} D \sigma\left(k T_{s}\right)
$$

If (11) is solved for the equivalent control, the following is obtained

$$
u_{e q}\left(k T_{s}\right)=u\left(k T_{s}\right)+(G B)^{-1}\left(\frac{\sigma\left((k+1) T_{s}\right)-\sigma\left(k T_{s}\right)}{T_{s}}\right)
$$

Since the system is causal, and control cannot be dependent on a future value of $\sigma$, the only way to estimate the current value of the equivalent control is by approximating by a single-step backward value computed from (14) as follows,

$$
\hat{u}_{e q_{k}} \cong u_{e q_{k-1}}=u_{k-1}+(G B)^{-1}\left(\frac{\sigma_{k}-\sigma_{k-1}}{T_{s}}\right)
$$

Here $\hat{u}_{e q_{k}}\left(\right.$ or $\left.\hat{u}_{e q}\left(k T_{s}\right)\right)$ is the estimate of the current value of the equivalent control. If (14) is inserted in (12)

$$
u_{k}=u_{k-1}+(G B)^{-1}\left(D \sigma_{k}+\frac{\sigma_{k}-\sigma_{k-1}}{T_{s}}\right)
$$

It easily seen that the above control law is derived from discrete-time approximations based on the continuoustime equations. Hence, it must be shown that the above control satisfies the original conditions based on which it was designed. These conditions are the Lyapunov condition and existence of Sliding Mode.

\section{B. Closed-Loop Behavior with the Approximated Control}

As a consequence of the approximations that were made in the derivation of the discrete-time control law some deviations in the sliding surface from the desired sliding manifold may exist. This deviation of the sliding surface from the desired manifold at each sampling instant will be analyzed. Analysis of the inter-sampling behavior of the sliding surface will also be analyzed. Considering (1), the derivative of the sliding surface is given by

$$
\dot{\sigma}(t)=G\left(\dot{x}^{r}-\dot{x}\right)=G \dot{x}^{r}-G f-G B u(t)
$$

The discrete-time equivalent of the sliding manifold can be obtained by taking the integral on both sides of (16) from $k T_{s}$ to $(k+1) T_{s}$ 


$$
\sigma_{k+1}-\sigma_{k}=\int_{k T_{s}}^{(k+1) T_{s}}\left(G \dot{x}^{r}-G f-G B u(t)\right) d t
$$

Applying a sample and hold to the control input between consecutive samples $u(t)=u_{k}$ for $k T_{s} \leq t<(k+1) T_{s}$

$$
\sigma_{k+1}-\sigma_{k}=\int_{k T_{s}}^{(k+1) T_{s}}\left(G \dot{x}^{r}-G f\right) d t-T_{s} G B u_{k}
$$

Using the assumptions that $\dot{x}^{r}$ and $f$ are smooth and bounded, the integrations in (18) can be approximated by using Euler's integration

$$
\sigma_{k+1}=\sigma_{k}+T_{s} G\left(\dot{x}_{k}^{r}-f_{k}\right)-T_{s} G B u_{k}+O\left(T_{s}{ }^{2}\right)
$$

Here $O\left(T_{s}^{2}\right)$ is the error introduced due to Euler's integration, [3]. If the control defined by (15) is introduced into (19)

$$
\begin{gathered}
\sigma_{k+1}=\sigma_{k}+T_{s} G\left(\dot{x}_{k}^{r}-f_{k}\right) \\
-T_{s} G B u_{k-1}-T_{s} D \sigma_{k}-\sigma_{k}+\sigma_{k-1}+O\left(T_{s}{ }^{2}\right)
\end{gathered}
$$

After some simplifications (20) is reduced to

$$
\begin{aligned}
\sigma_{k+1}= & T_{s} G\left(\dot{x}_{k}^{r}-f_{k}\right)-T_{s} G B u_{k-1} \\
& -T_{s} D \sigma_{k}+\sigma_{k-1}+O\left(T_{s}{ }^{2}\right)
\end{aligned}
$$

If $T_{s} G\left(\dot{x}_{k-1}^{r}-f_{k-1}\right)$ is added and subtracted from the r.h.s of (21), the following is obtained

$$
\begin{array}{r}
\sigma_{k+1}=T_{s} G\left(\dot{x}_{k}^{r}-f_{k}\right)-T_{s} G\left(\dot{x}_{k-1}^{r}-f_{k-1}\right)-T_{s} D \sigma_{k} \\
+\underbrace{T_{s} G\left(\dot{x}_{k-1}^{r}-f_{k-1}\right)-T_{s} G B u_{k-1}}_{\sigma_{k}-\sigma_{k-1}+O\left(T_{s}^{2}\right)}+\sigma_{k-1}+O\left(T_{s}^{2}\right)
\end{array}
$$

After some simplifications, (22) becomes

$$
\sigma_{k+1}=\sigma_{k}-T_{s} D \sigma_{k}+T_{s} G\left(\Delta \dot{x}_{k}^{r}-\Delta f_{k}\right)+O\left(T_{s}^{2}\right)
$$

Here $\Delta \dot{x}_{k}^{r}=\dot{x}_{k}^{r}-\dot{x}_{k-1}^{r}$ and $\Delta f_{k}=f_{k}-f_{k-1}$. Note that if $D=1 / T_{s}$, then the r.h.s of (23) is of order $O\left(T_{s}^{2}\right)$, keeping in mind that $\dot{x}^{r}$ and $f$ are smooth and continuous. Hence,

$$
\sigma_{k+1}=O\left(T_{s}^{2}\right)
$$

Hence, it is shown that the maximum deviation from the sliding surface at each sampling instant is of order $O\left(T_{s}^{2}\right)$.

Next, it will be shown that the inter-sampling deviation of the sliding surface from the desired manifold is also of order $O\left(T_{s}^{2}\right)$.

Consider the inter-sampling instant of $t=k T_{s}+\tau$ where $0 \leq \tau \leq T_{s}$. If (16) is integrated on both sides from $k T_{s}$ to $k T_{s}+\tau$

$$
\sigma\left(k T_{s}+\tau\right)-\sigma_{k}=\int_{k T_{s}}^{k T_{s}+\tau}\left(G \dot{x}^{r}-G f-G B u(t)\right) d t
$$

Applying the sample and hold to the control and Euler's integration to the remaining integral gives

$$
\sigma\left(k T_{s}+\tau\right)=\sigma_{k}+\tau G\left(\dot{x}_{k}^{r}-f_{k}\right)-\tau G B u_{k}+O\left(\tau^{2}\right)
$$

If the control defined by (15) is introduced into (26)

$$
\begin{gathered}
\sigma\left(k T_{s}+\tau\right)=\sigma_{k}+\tau G\left(\dot{x}_{k}^{r}-f_{k}\right)-\tau G B u_{k-1} \\
-\tau D \sigma_{k}-\frac{\tau}{T_{s}}\left(\sigma_{k}-\sigma_{k-1}\right)+O\left(\tau^{2}\right)
\end{gathered}
$$

If $\tau G\left(\dot{x}_{k-1}^{r}-f_{k-1}\right)$ is added and subtracted from the r.h.s of (21) and $D=1 / T_{s}$, the following is obtained

$$
\begin{gathered}
\sigma\left(k T_{s}+\tau\right)=\sigma_{k}+\frac{\tau}{T_{s}} G\left(T_{s}\left(\Delta \dot{x}_{k}^{r}-\Delta f_{k}\right)\right)-\frac{\tau}{T_{s}} \sigma_{k}-\frac{\tau}{T_{s}} \sigma_{k} \\
+\frac{\tau}{T_{s}} \underbrace{G\left(T_{s}\left(\dot{x}_{k-1}^{r}-f_{k-1}\right)-T_{s} B u_{k-1}\right)}_{\sigma_{k}-\sigma_{k-1}+O\left(T_{s}^{2}\right)}+\frac{\tau}{T_{s}} \sigma_{k-1}+O\left(\tau^{2}\right)
\end{gathered}
$$

Further simplifications lead to

$$
\sigma\left(k T_{s}+\tau\right)=\sigma_{k}-\frac{\tau}{T_{s}} \sigma_{k}+\frac{\tau}{T_{s}} G\left(T_{s}\left(\Delta \dot{x}_{k}^{r}-\Delta f_{k}\right)\right)+O\left(\tau^{2}\right)
$$

If $\dot{x}^{r}$ and $f$ are smooth and continuous then

$$
\sigma\left(k T_{s}+\tau\right)=\sigma_{k}-\frac{\tau}{T_{s}} \sigma_{k}+O\left(\tau^{2}\right)
$$

Note that if $\sigma_{k}=O\left(T_{s}^{2}\right)$ as was shown previously then the maximum intersampling value of the sliding function is $O\left(T_{s}^{2}\right)$. Hence,

$$
\sigma\left(k T_{s}+\tau\right)=O\left(T_{s}^{2}\right)
$$




\section{Lyapunov Stability Analysis of the Closed-Loop System}

In this section it will be shown that with discrete-time control defined by (15) it is possible to satisfy the Lyapunov conditions (4) and (5) in discrete-time.

Starting with the definition of the Lyapunov function in discrete-time

$$
V_{k}=\sigma_{k}^{2}
$$

The difference of two consecutive values of the Lyapunov function in discrete-time can be given by

$$
V_{k+1}-V_{k}=\sigma_{k+1}^{2}-\sigma_{k}^{2}
$$

Here it is required that $V_{k+1}-V_{k}<0$ for $\sigma_{k} \neq 0$. However, it will be shown that $V_{k+1}-V_{k}<0$ for $\left|\sigma_{k}\right|>O\left(T_{s}^{2}\right)$. The condition $V_{k+1}-V_{k}<0$ means that

$$
\sigma_{k+1}^{2}-\sigma_{k}^{2}<0
$$

If (24) is inserted into (34),

$$
V_{k+1}-V_{k}=O\left(T_{s}^{4}\right)-\sigma_{k}^{2}
$$

Note that if $\left|\sigma_{k}\right|>O\left(T_{s}^{2}\right)$ then $V_{k+1}-V_{k}<0$. Thus, (35) shows that $\sigma_{k}$ is always converging towards a boundary of $O\left(T_{s}^{2}\right)$ around the desired sliding-manifold and (31) shows that once $\sigma_{k}$ reaches $O\left(T_{s}^{2}\right)$ boundary it will tend to stay in that boundary.

\section{ImPlementation ON A Piezo-STAGe}

In this work a piezo-stage that consists of a piezo-drive integrated with a sophisticated flexure structure for motion amplification is used. The flexure structure is wire-EDM-cut and is designed to have zero stiction and friction. Figure 1 shows the piezo-drive integrated flexure structure. In addition to the absence of internal friction, flexure stages exhibit high stiffness and high load capacity. Flexure stages are also insensitive to shock and vibration. However, since the piezo-drive exhibits nonlinear hysteresis behavior, the overall system will also exhibit the same behavior.

The dynamics of the piezo-stage can be represented by the following second-order differential equation coupled with hysteresis in the presence of external forces

$$
m_{e f f} \ddot{y}+c_{e f f} \dot{y}+k_{e f f} y=T(u(t)-h(y, u))-F_{e x t}
$$

Here $m_{\text {eff }}$ denotes the effective mass of the stage, $y$ denotes the displacement of the stage, $c_{\text {eff }}$ denotes the effective damping of the stage, $k_{\text {eff }}$ denotes the effective stiffness of the stage, $T$ denotes the electromechanical transformation ratio, $u$ denotes the input voltage and $h(y, u)$ denotes the non-linear hysteresis that has been found to be a function of $y$ and $u$, [1], and $F_{\text {ext }}$ is the external force acting on the stage.

The structure of model (36) is showing that, from the mechanical motion the hysteresis may be perceived as a disturbance force that satisfy matching conditions. This means that the sliding mode based control should be able to reject the influence of the hysteresis nonlinearity on the mechanical motion. At the same time it is obvious that the lumped disturbance consisting of the external force acting on the system and the hysteresis can be estimated, thus allowing the application of the disturbance rejection method in the overall system design.

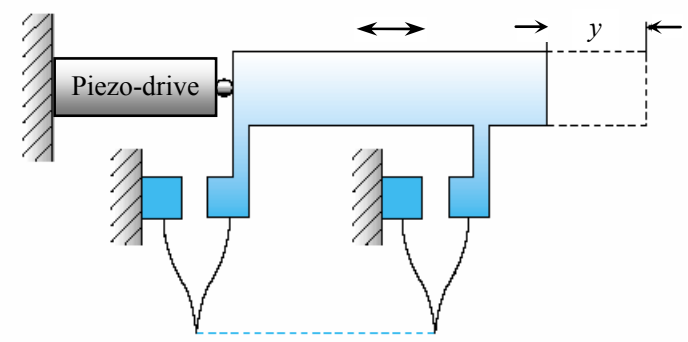

Figure 1. Fig. 1. Structure of a flexure piezo-stage

To facilitate the writing of the control law, (36) is written into the state-space form

$$
\begin{aligned}
& \dot{x}_{1}=\dot{y}=x_{2} \\
& \dot{x}_{2}=\ddot{y}=-\frac{k_{\text {eff }}}{m_{\text {eff }}} x_{1}-\frac{c_{\text {eff }}}{m_{\text {eff }}} x_{2}+\frac{T}{m_{\text {eff }}} u-\frac{T}{m_{\text {eff }}} h(y, u)-\frac{F_{\text {ext }}}{m_{\text {eff }}}
\end{aligned}
$$

From here it can be seen that the input matrix is

$$
B=\left\{\begin{array}{ll}
0 & \frac{T}{m_{\text {eff }}}
\end{array}\right\}^{T}
$$

The matrix $G$ for this case will be selected to be

$$
G=\{\lambda \quad 1\}
$$

Here $\lambda$ is a positive constant. Hence, the controller will be in the following form

$$
u_{k}=u_{k-1}+\frac{m_{e f f}}{T}\left(D \sigma_{k}+\frac{\sigma_{k}-\sigma_{k-1}}{T_{s}}\right)
$$

The results that it will be shown in this section are for the case of SMC and for comparison purposes PID results 
will also be shown. Figures 2 and 3 depict the tracking of the piezo-stage for a $0.25 \mathrm{~Hz}$ sinusoidal reference.

\section{DistURBANCE OBSERVER}

\section{A. Design and Analysis of the Disturbance Observer}

The structure of the observer is based on (36) under the assumption that all the plant parameter uncertainties, nonlinearities and external disturbances can be represented as a lumped disturbance. As it is obvious, $y$ is the displacement of the plant and is measurable. Likewise, $u(t)$ is the input to the plant and is also measurable. Hence, the nominal structure of the plant is defined as follows

$$
\begin{gathered}
m_{N} \ddot{y}+c_{N} \dot{y}+k_{N} y=T_{N} u(t)-F_{d} \\
F_{d}=T_{N} h+\Delta T\left(v_{i n}+v_{h}\right)+\Delta m \ddot{y}+\Delta c \dot{y}+\Delta k y
\end{gathered}
$$

Here $m_{N}, c_{N}, k_{N}$ and $T_{N}$ are the nominal plant parameters while $\Delta m, \Delta c, \Delta k$ and $\Delta T$ are the uncertainties of the plant parameters. Since $y$ and $u(t)$ are measured the proposed observer is of the following form

$$
m_{N} \ddot{\hat{y}}+c_{N} \dot{\hat{y}}+k_{N} \hat{y}=T_{N} u-T_{N} u_{c}
$$

Here $\hat{y}$ is the estimated position $u$ is the plant control input and $u_{c}$ is the observer control input. If $\hat{y}$ can be forced to track $y$ then obviously $F_{d}=T_{N} u_{c}$. The observer controller that is used is in the SMC framework. Selecting the following sliding manifold

$$
\sigma_{o b s}=\lambda_{o b s}(y-\hat{y})+(\dot{y}-\dot{\hat{y}})
$$

here $\lambda_{o b s}$ is a positive constant. If $\sigma_{o b s}$ is forced to zero then $\hat{y}$ is forced to track $y$. The controller used is

$$
u_{c_{k}}=u_{c_{k-1}}+\frac{m_{e f f}}{T}\left(D_{o b s} \sigma_{o b s_{k}}+\frac{\sigma_{o b s_{k}}-\sigma_{o b s_{k-1}}}{T_{s}}\right)
$$

The frequency response of the disturbance observer output with respect to the disturbance is depicted in the Fig. 4. The response shown is for the case when the sampling time is $100 \mu \mathrm{s}$ and the controller parameters being $D_{o b s}=\lambda_{\text {obs }}=1 / T_{s}$.
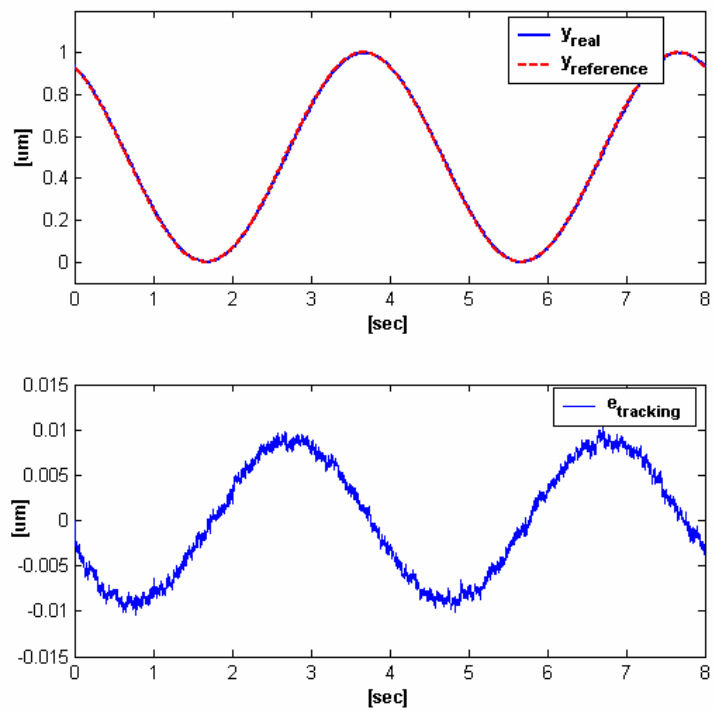

Figure 2. Fig. 2. Sinusoidal reference tracking with SMC
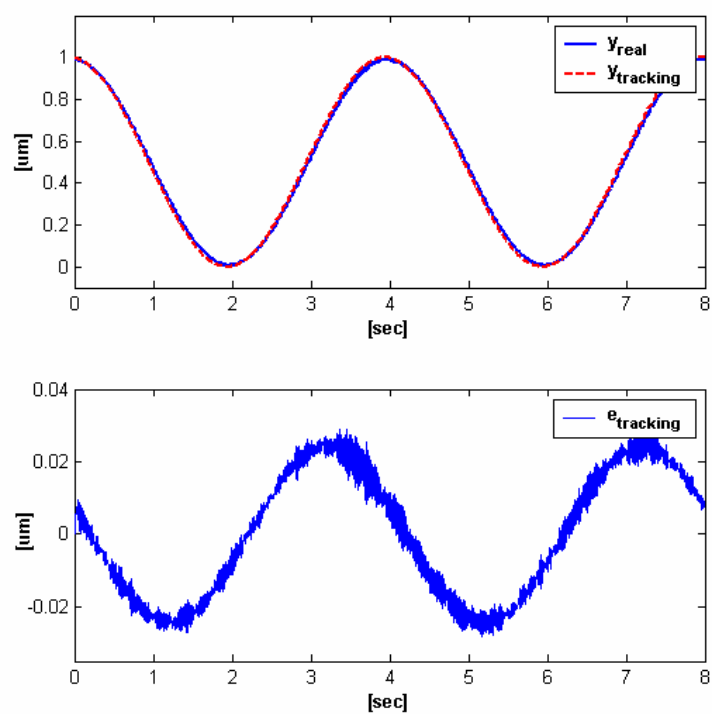

Figure 3. Fig. 3. Sinusoidal reference tracking with PID controller

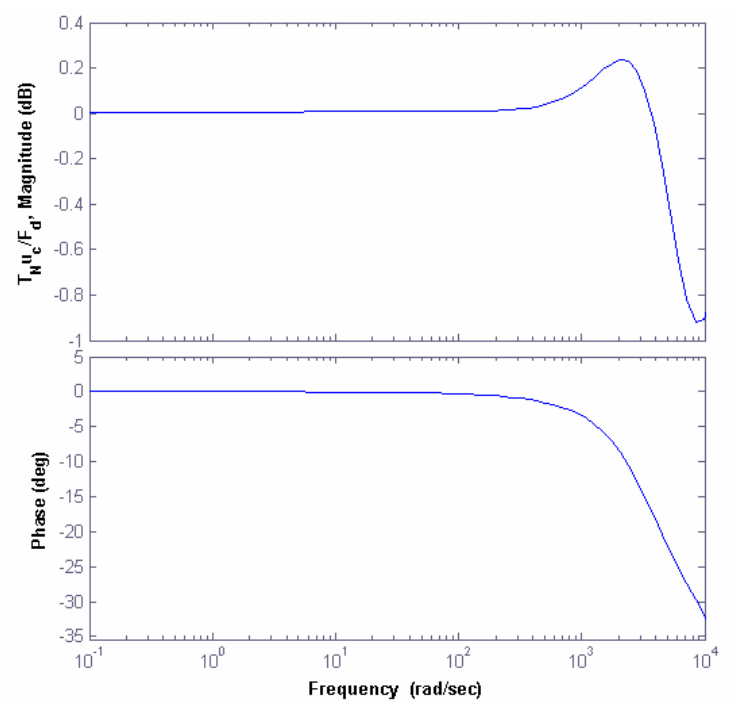

Figure 4. Fig. 4. Magnitude and Phase Plots of the Observer Response 


\section{B. Closed-Loop Experiments with Disturbance Compensation}

The disturbance observer shown above was implemented with closed-loop control. The observer implementation is depicted in the Fig. 5. The experiments show a notable improvement in tracking for the cases of Sliding mode controller and PID controller.

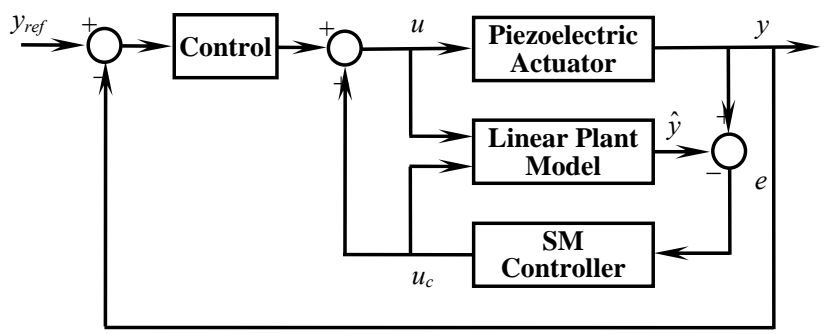

Figure 5. Fig. 5. Closed-Loop control with disturbance compensation
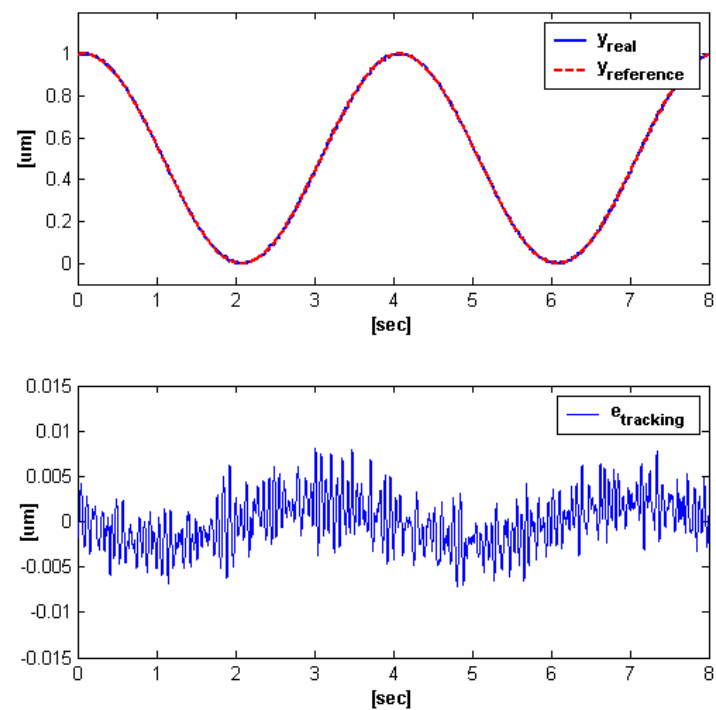

Figure 6. Fig. 6. Sinusoidal reference tracking with the SMC and disturbance compensation
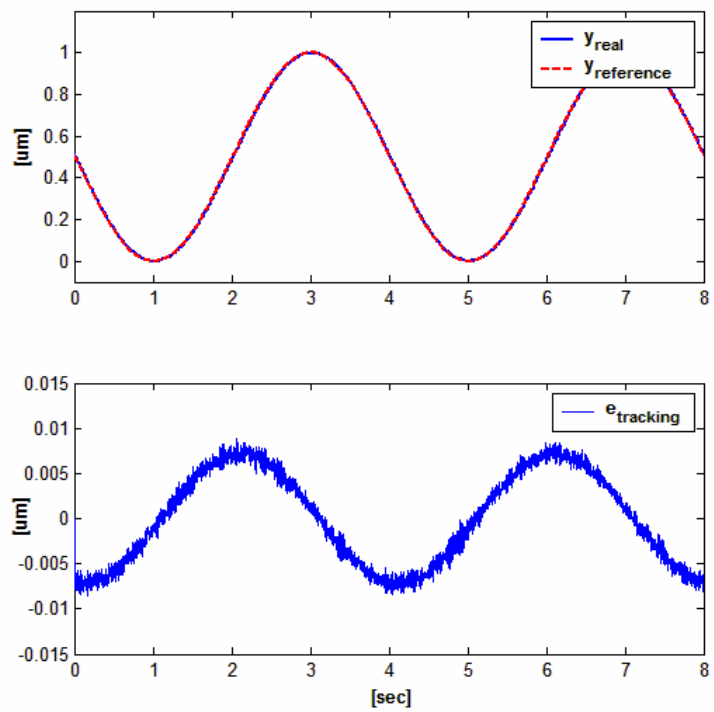

Figure 7. Fig. 7. Sinusoidal reference tracking with PID and disturbance compensation

\section{CONCLUSION}

In this paper the robustness of a designed discrete-time Sliding mode controller was shown. It was also shown that the controller can push the states of the system to an $O\left(T_{s}^{2}\right)$ boundary around the desired sliding manifold. Experiments were also conducted to show the effectiveness of the controller. As an extension, it was shown that the inclusion of disturbance compensation via disturbance observer can improve the overall closed-loop system.

\section{REFERENCES}

[1] [1] Wu-Chung Su, S. V. Drakunov, \& Umit Ozguner, An O(T²) Boundary Layer in Sliding Mode for Sampled-Data Systems, IEEE Transactions on Automatic Control, 45(3), 2000, 482-485.

[2] [2] Michael Goldfarb \& Nikola Celanovic, Modeling Piezoelectric Actuators for Control of Micromanipulation, IEEE Control Systems Magazine, 7(2), 1997, 69-79.

[3] [3] S. C. Chapra, \& R. P. Canale, Numerical methods for engineers (Singapore: WCB/McGraw-Hill, 1998 